\title{
Cost-Effectiveness of Orthogeriatric and Fracture Liaison Service Models of Care for Hip Fracture Patients: A Population-Based Study
}

\author{
Jose Leal, ${ }^{1}$ Alastair M Gray, ${ }^{1}$ Samuel Hawley, ${ }^{2}$ Daniel Prieto-Alhambra, ${ }^{2,3,4}$ Antonella Delmestri, ${ }^{2}$ \\ Nigel K Arden, ${ }^{2,3}$ Cyrus Cooper, ${ }^{2,3}$ M Kassim Javaid, ${ }^{2,3}$ Andrew Judge ${ }^{2,3}$; and the REFReSH Study Group \\ ${ }^{1}$ Health Economics Research Centre, Nuffield Department of Population Health, University of Oxford, Oxford, UK \\ ${ }^{2}$ Oxford NIHR Musculoskeletal Biomedical Research Unit, Nuffield Department of Orthopaedics, Rheumatology, and Musculoskeletal Sciences, \\ University of Oxford, Oxford, UK \\ ${ }^{3}$ MRC Lifecourse Epidemiology Unit, University of Southampton, Southampton General Hospital, Southampton, UK \\ ${ }^{4}$ GREMPAL Research Group (Idiap Jordi Gol) and Musculoskeletal Research Unit (Fundació IMIM-Parc Salut Mar), Universitat Autònoma de \\ Barcelona, Barcelona, Spain
}

\begin{abstract}
Fracture liaison services are recommended as a model of best practice for organizing patient care and secondary fracture prevention for hip fracture patients, although variation exists in how such services are structured. There is considerable uncertainty as to which model is most cost-effective and should therefore be mandated. This study evaluated the cost-effectiveness of orthogeriatric (OG)- and nurse-led fracture liaison service (FLS) models of post-hip fracture care compared with usual care. Analyses were conducted from a health care and personal social services payer perspective, using a Markov model to estimate the lifetime impact of the models of care. The base-case population consisted of men and women aged 83 years with a hip fracture. The risk and costs of hip and non-hip fractures were derived from large primary and hospital care data sets in the UK. Utilities were informed by a metaregression of 32 studies. In the base-case analysis, the orthogeriatric-led service was the most effective and cost-effective model of care at a threshold of $£ 30,000$ per quality-adjusted life years gained (QALY). For women aged 83 years, the OG-led service was the most cost-effective at $£ 22,709 / \mathrm{QALY}$. If only health care costs are considered, OG-led service was cost-effective at $£ 12,860 / \mathrm{QLLY}$ and $£ 14,525 / \mathrm{QALY}$ for women and men aged 83 years, respectively. Irrespective of how patients were stratified in terms of their age, sex, and Charlson comorbidity score at index hip fracture, our results suggest that introducing an orthogeriatrician-led or a nurse-led FLS is cost-effective when compared with usual care. Although considerable uncertainty remains concerning which of the models of care should be preferred, introducing an orthogeriatrician-led service seems to be the most cost-effective service to pursue. @ 2016 American Society for Bone and Mineral Research.
\end{abstract}

KEY WORDS: COST-EFFECTIVENESS; OSTEOPOROSIS; HIP FRACTURE; SECONDARY PREVENTION; FRACTURE LIAISON SERVICE; NATURAL EXPERIMENT; HEALTH ECONOMICS; COST-UTILITY; HIP FRACTURE; COST

\section{Introduction}

$\mathrm{H}$ ip fractures are a major public health problem, with high morbidity, mortality, and health and social care costs. ${ }^{(1-3)}$ In the UK, hip fractures account for $£ 1.1$ billion per year in hospital costs, ${ }^{(4)}$ which is expected to rise to $£ 1.5$ billion by $2025^{(4)}$ when 104,000 annual cases are predicted. ${ }^{(3)}$ A previous study showed that acute hospital admission resulting from hip fracture was the largest component of hospital costs, with higher costs and length of stay for second compared with index hip fractures. ${ }^{(4)}$ There is therefore a strong economic incentive to identify and implement cost-effective measures for the provision of timely patient care and secondary fracture prevention after an index hip fracture.

Fracture liaison services are recommended as a model of best practice for patients with hip fracture. This is supported by international guidance ${ }^{(5,6)}$ patient organizations, the UK Department of Health, ${ }^{(7,8)}$ and several UK national bodies (British Orthopaedic Association, ${ }^{(9)} \mathrm{NICE}_{1}^{(10)}$ National Osteoporosis Society, and Age UK $\left.{ }^{(11)}\right)$. The proposed model consists of two main stages of care: 1) orthogeriatric services focusing on achieving optimal recovery after hip fracture, and 2) nurse-led fracture liaison services focusing on secondary fracture prevention of fragility fractures.

Received in original form June 8, 2016; revised form September 6, 2016; accepted September 14, 2016. Accepted manuscript online Month 00, 2016. Address correspondence to: Jose Leal, DPhil, Health Economics Research Centre, University of Oxford, Old Road Campus, OX3 7LF, Oxford, UK. E-mail: Jose.leal@dph.ox.ac.uk

Additional Supporting Information may be found in the online version of this article.

Journal of Bone and Mineral Research, Vol. xx, No. xx, Month 2016, pp 1-9

DOI: 10.1002/jbmr.2995

(C) 2016 American Society for Bone and Mineral Research 
The Glasgow Fracture Liaison Service ${ }^{(12)}$ reported that for every 1000 patients with a fragility fracture assessed by that FLS, 18 fragility fractures (including 11 hip fractures) were prevented. However, no firm evidence or evidence-based consensus exists as to which care model should be mandated across the NHS. As a result, current practice reflects significant variations across NHS hospital providers in the adoption and organization of FLS. ${ }^{(13)}$

We used large health care data sets based on deidentified computerized records, together with a detailed evaluation of hospital hip fracture services in a UK region, ${ }^{(14)}$ to estimate the "real-world" impact of the different models of care in terms of morbidity, survival, and costs, and to determine the costeffectiveness of orthogeriatric (OG)- and nurse-led fracture liaison service (FLS) models of care after hip fracture compared with usual care in the English NHS.

\section{Materials and Methods}

\section{Decision model and models of care}

We estimated the cost-effectiveness of the following models of care for all patients with a hip fracture admitted to an NHS hospital: 1) Introduction of an orthogeriatrician-led (OG) model of post-hip fracture care; 2 ) Introduction of a nurse-led fracture liaison (FLS) model of post-hip fracture care; 3) Standard posthip fracture care.

These models of care reflect the services provided in one regional area in the UK that comprises 11 hospitals receiving patients with acute hip fractures. Details on the OG and FLS models delivered within the region from 2003 to 2013 have been published elsewhere. ${ }^{(14,15)}$ Briefly, although there was variation across hospitals, the introduction of the OG model involved the appointment of an orthogeriatrician as the clinical lead, responsible for case finding, preoperative assessment, patient assessment, and treatment initiation, as well as having involvement in postoperative care. The average staff level of an OG for secondary fracture prevention services was estimated at 0.75 whole time equivalents (WTE) within region. The FLS model involved the appointment of a nurse specialist (osteoporosis or trauma) responsible for case finding, assessment, treatment recommendations, medication assessment, and preparation of the follow-up plan, as well as for providing additional support for management of bone health in hospital. In this region of the UK, treatment adherence monitoring was predominately delegated to primary care. Finally, standard care post-hip fracture care reflects care provided without the introduction and/or expansion of OG and FLS models of care.

The perspective adopted was that of the NHS in England and personal social services, including primary and secondary health care and care home costs. Primary care costs included GP and practice nurse contacts, visits to other community health care professionals (eg, health visitor, physiotherapist), laboratory tests, and drugs. Secondary health care costs included outpatient visits, accident and emergency contacts, day cases, and inpatient admissions. Primary and secondary care costs captured hip fracture-related costs as well as all other costs. We did not include the cost of walking aids, home adaptation costs, or home care costs funded by councils or local organizations (eg, live-in help, meals, nursing care, domestic help, etc.).

Given the natural history of hip fracture progression with recursive events, a Markov model was built to evaluate the lifetime costs (quality-adjusted) life expectancy and costeffectiveness. The model structure was defined using an iterative process involving discussions with clinical experts and epidemiologists involved in the REFRESH study and supplemented by a literature review of economic models in the disease area.

The Markov model, developed in Excel (Microsoft, Redmond, WA, USA), was used to simulate the natural history of individuals after an index hip fracture across health states representing: history of index hip fracture, second hip fracture; major non-hip fracture(s) (pelvic, spine, wrist, humerus, and rib) requiring hospitalization; living in patient's own home or in a care home; and dead (within 30 days post-hip fracture or within year) (Fig. 1 and Supplemental Fig. S1). We assumed that if a patient transitioned to a care home they would not go back to their own home for the remainder of their life. A cycle length of one year was considered appropriate given the natural history of hip fracture patients, and half-cycle correction was performed. ${ }^{(16)}$ All costs and outcomes were discounted using the recommended annual rate of $3.5 \%$. Costs are quoted in 2012/2013 prices.

\section{Derivation of model inputs}

\section{Study subjects in Hospital Episode Statistics (HES) data set}

We extracted all hospital records for 33,152 patients older than 60 years who had had an emergency hospital admission with a primary ICD-10 diagnosis code for hip fracture (S72.0, S72.1, S72.2, and S72.9) between April 2003 and March 2013 for a representative region of the UK (Fig. 2 and Supplemental Table S1). ${ }^{(4)}$ The HES data were used to develop the risk equations for the following events: time to second hip fracture, time to major non-hip fragility fracture requiring hospitalization, discharge to care home (nursing or residential) after hip fracture, and time to death. The data were also used to estimate the annual hospitalization costs for each health state of the model. See Supplemental Material and Leal and colleagues ${ }^{(4)}$ for details on the valuation of hospital resource use.

Study subjects in the Clinical Practice Research Datalink (CPRD) data set

We extracted all primary care contacts, laboratory tests, and prescribed drugs for 4063 patients registered in the CPRD GOLD database between April 1, 2003, and March 31, 2012, who had linked hospital use records indicating a hip fracture (Fig. 2 and Supplemental Table S2). Hip fracture was identified in primary care records using predefined READ codes (Supplemental Table S3). The data set was used to estimate the annual primary care costs for each health state of the model. Resource use was valued using national cost databases for the year 2012/13 $3^{(17-19)}$ (see Supplemental Table S4 for more details).

\section{Relative effectiveness of OG and FLS}

Previous work using the HES cohort ${ }^{(15)}$ informed the relative effectiveness of the models of care measured by time to second hip fracture (hazard ratio [HR]) and time to death (HR) adjusted for confounding factors such as age, sex, deprivation, and Charlson comorbidity index (Table 1). In the base case, we assumed the effect of introducing OG or FLS relative to usual care on mortality after hip fracture was present only for the index fracture and in the year of the fracture, and the effect on second hip fracture was present only in the first 2 years after index hip fracture. These assumptions were explored in sensitivity analysis. 


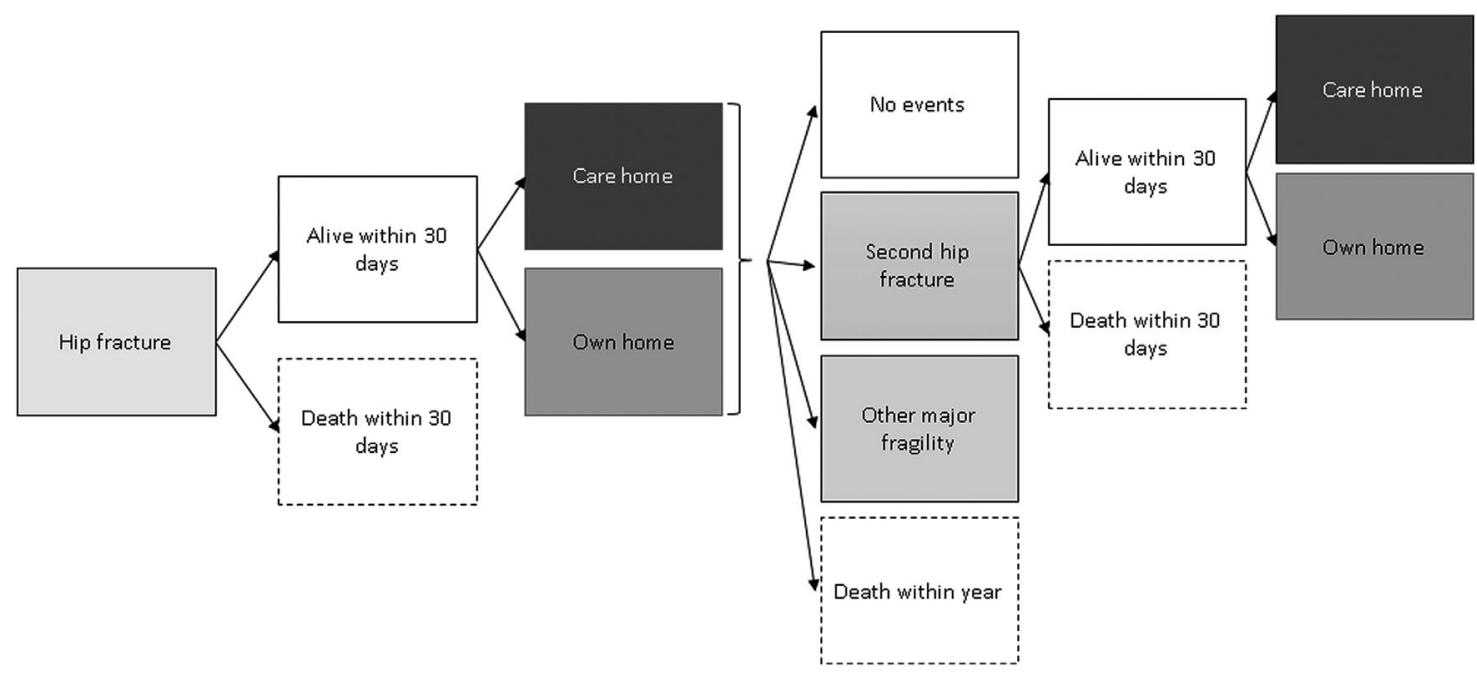

Fig. 1. Model structure and allowed transitions in the first year of simulation. The model simulated the transition of a cohort of patients with an index hip fracture through the health states over time to estimate expected costs and outcomes. At the start of the simulation, patients with a hip fracture could die within 30 days or be discharged home or to a care home (nursing or residential care home). In the same cycle, patients could then develop a second hip fracture, other major fragility fracture requiring hospitalization (non-hip such as pelvic, spine, wrist, humerus, and rib), have no further events, or die. If patients experienced a second hip fracture, they could die within 30 days or, if alive, be discharged to a care home or their own home.

\section{Cost of introducing OG and FLS}

Based on the survey of 11 hospitals in the UK region ${ }^{(14)}$ reporting staffing levels and non-clinical activities (eg, clinical leadership roles) of an orthogeriatrician and a nurse leading the FLS within the same region, an FLS was assumed to operate at $100 \%$ capacity (whole time equivalent [WTE]), whereas an OG was assumed to operate at $75 \%$ capacity. The annual costs of an OG and an FLS per hip fracture patient were estimated by multiplying the respective WTE by the total annual costs ${ }^{(18)}$ and dividing these by 450 hip fracture patients (average patients seen per year across the 11 hospitals in the survey ${ }^{(20)}$ ) (Table 1).
Clinical Practice Research Datalink (CPRD) dataset

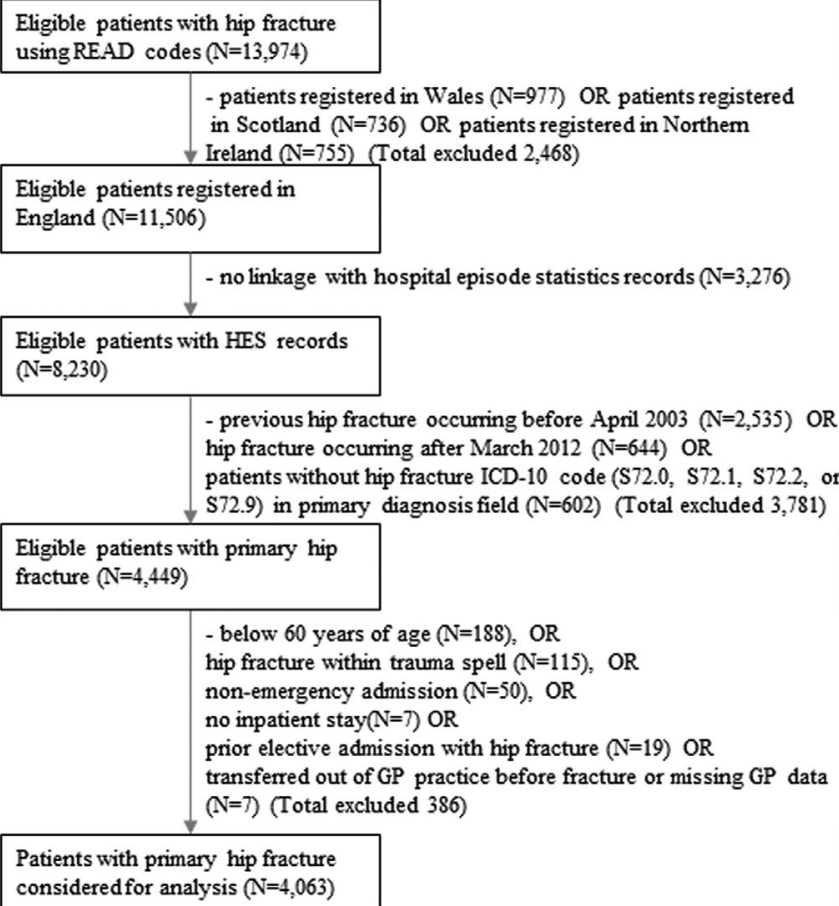

- below 60 years of age $(N=188), O R$ hip fracture within trauma spell $(\mathrm{N}=115), \mathrm{OR}$ non-emergency admission $(\mathrm{N}=50)$, OR no inpatient stay $(\mathrm{N}=7) \mathrm{OR}$

prior elective admission with hip fracture $(\mathrm{N}=19) \mathrm{OR}$ transferred out of GP practice before fracture or missing GP data $(\mathrm{N}=7$ ) (Total excluded 386)

Patients with primary hip fracture considered for analysis $(\mathrm{N}=4,063)$

Fig. 2. Identification of patients with hip fracture in the HES and CPRD data sets.
Hospital Episode Statistics (HES) dataset

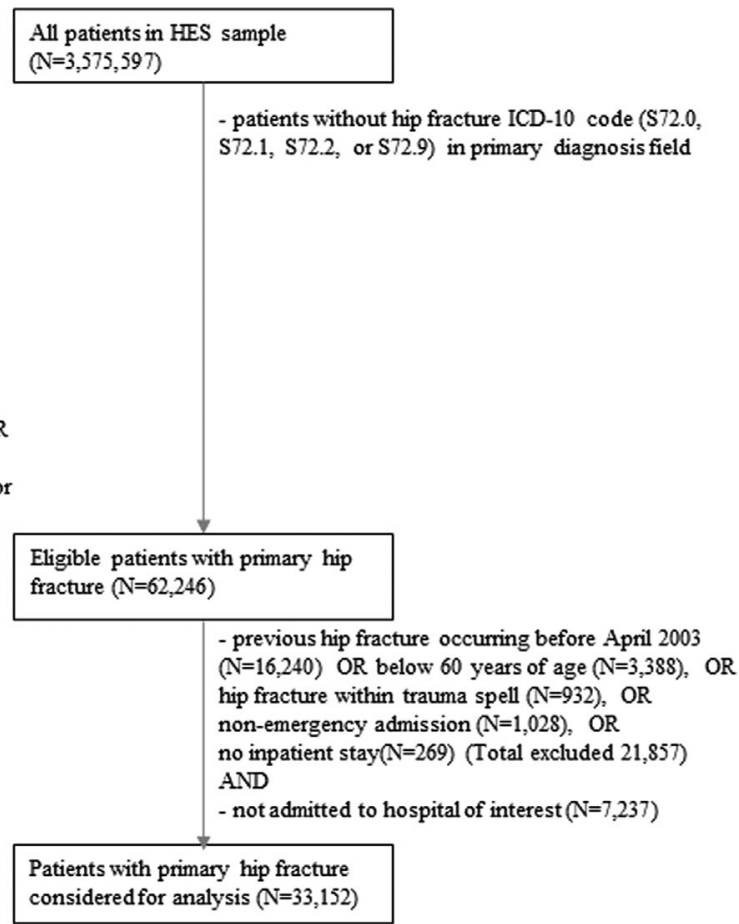




\begin{tabular}{|c|c|c|}
\hline Parameter & Mean $(95 \% \mathrm{Cl})$ & Source \\
\hline \multicolumn{3}{|l|}{ Relative effectiveness (hazard ratio) } \\
\hline \multicolumn{3}{|l|}{ Introducing OG relative to usual care } \\
\hline 30-day mortality after index hip fracture & $0.73(0.65-0.82)$ & Hawley ${ }^{(15)}$ \\
\hline 1-year mortality after index hip fracture & $0.81(0.75-0.87)$ & Hawley ${ }^{(15)}$ \\
\hline 2-year risk of developing second hip fracture & $0.95(0.79-1.15)$ & Hawley ${ }^{(15)}$ \\
\hline \multicolumn{3}{|l|}{ Introducing FLS relative to usual care } \\
\hline 30-day mortality after index hip fracture & $0.80(0.71-0.91)$ & Hawley ${ }^{(15)}$ \\
\hline 1-year mortality after index hip fracture & $0.84(0.77-0.93)$ & Hawley ${ }^{(15)}$ \\
\hline 2-year risk of developing second hip fracture & $1.03(0.85-1.26)$ & Hawley ${ }^{(15)}$ \\
\hline $\begin{array}{l}\text { Discharge to care home after index hip fracture } \\
\text { (previously not in care home) }\end{array}$ & Supplemental Table S8 & HES \\
\hline $\begin{array}{l}\text { Discharge to care home after second hip fracture } \\
\text { (previously not in care home) }\end{array}$ & Supplemental Table S8 & HES \\
\hline Risk of second hip fracture & Supplemental Table S9 & HES \\
\hline Risk of major non-hip fracture requiring hospitalization & Supplemental Table S9 & HES \\
\hline 30-day mortality after index hip fracture & Supplemental Table S9 & HES \\
\hline 30-day mortality after second hip fracture & Supplemental Table S9 & HES \\
\hline All-cause mortality post 30 days of fracture & Supplemental Table S9 & HES \\
\hline \multicolumn{3}{|l|}{ Intervention $\operatorname{cost}^{\mathrm{a}}$} \\
\hline Fracture liaison nurse (grade 7) per hip fracture patient & $£ 200$ & Curtis $^{(18)}$ \\
\hline Orthogeriatrician (consultant) & $£ 420$ & Curtis $^{(18)}$ \\
\hline $\begin{array}{l}\text { Proportion discharged to a care home that go to a } \\
\text { nursing home }\end{array}$ & $0.48(0.47-0.49)$ & $\begin{array}{l}\text { National Hip Fracture } \\
\text { Database }^{(20)}\end{array}$ \\
\hline Primary care costs in year of index fracture & Supplemental Table S10 & CPRD \\
\hline Primary care costs in years after index fracture & Supplemental Table S10 & CPRD \\
\hline Hospitalization costs in year of index hip fracture & Supplemental Table S11 & HES \\
\hline Hospitalization costs in year of second hip fracture & Supplemental Table S11 & HES \\
\hline Hospitalization costs in years after fracture & Supplemental Table S11 & HES \\
\hline Hospitalization costs if non-hip fracture occurs & Supplemental Table S12 & HES \\
\hline Hospitalization costs if death occurs & Supplemental Table S12 & HES \\
\hline \multicolumn{3}{|l|}{ Cost of institutionalization (per year) ${ }^{b}$} \\
\hline Nursing home & $£ 39,000$ & Curtis $^{(18)}$ \\
\hline Residential home & $£ 27,664$ & Curtis $^{(18)}$ \\
\hline \multicolumn{3}{|l|}{ Utility of hip fracture patients } \\
\hline Within 1 month of index fracture & $0.46(0.38-0.55)$ & Lit review \\
\hline At 12 months & $0.53(0.47-0.61)$ & Lit review \\
\hline At 24 months and after & $0.66(0.60-0.74)$ & Lit review \\
\hline Discount rate for costs and outcomes & $3.5 \%$ & HM Treasury \\
\hline
\end{tabular}

$\mathrm{HES}=$ Hospital Episode Statistics database; $\mathrm{CPRD}=$ Clinical Practice Research Datalink database.

ancludes salary, salary oncosts, qualification costs, management and non-staff overheads, and capital overheads.

${ }^{b}$ Nursing home at $£ 39,000$ per year ( $£ 750$ per week times 52 weeks) and residential home at $£ 27,664$ per year ( $£ 532$ per week times 52 weeks).

The OG- and FLS-specific costs were assumed to occur in the year of the index hip fracture, which was explored in sensitivity analysis.

\section{Statistical analysis}

The rate of second hip fracture or of major non-hip fracture requiring hospitalization after the index fracture was estimated using parametric survival models. Time to event was determined in continuous time from the onset of first hip fracture, using the censor date of death or the date of administrative censoring (March 31, 2013). All-cause mortality was derived using two logistic models to capture the high mortality in the first 30 days after first and second hip fracture (separate models) and a Gompertz proportional hazards survival model for the subsequent years. Time to death was modeled in continuous time, using each patient's current age as time at risk to better extrapolate beyond the observed follow-up period. ${ }^{(21)}$ Logistic models estimated the probability of admission to care home (nursing or residential) after hip fracture (index and second). A generalized linear model (GLM), with a gamma distribution and identity link function, was used to predict the annual primary and hospital care costs by health state. Time-invariant covariates included sex, age, and Charlson comorbidity index at first hip fracture, and timevariant factors included occurrence or history of second hip fracture, occurrence or history of major non-hip fracture, and admission from care home or own home. The proportion of those discharged to a care home that go to a nursing home was derived from the National Hip Fracture Database report ${ }^{(20)}$ and costed using a national cost database ${ }^{(18)}$ (Table 1). Statistical analysis was carried out using Stata v. 12 (StataCorp, College Station, TX, USA). See Supplemental Material for more methodological details. 


\section{Quality of life in patients with hip fracture}

We used a meta-regression approach, with a linear mixed-effects model, to synthesize absolute utility data from 32 studies $(21,085$ patients) identified by literature review, which reported preference-based quality of life for patients with hip fracture (eg, EQ-5D, EQVAS, HUI2, etc.) (Supplemental Fig. S2 and Supplemental Table S5). The resulting model was used to predict the EQ-5D utility values of hip fracture patients at several time points (onset and 1, 6, 12, 18, 24 months) to estimate utility up to 2 years after hip fracture using the area under the curve (Table 1). We assumed that utilities remained constant after the first year after hip fracture (ie, 0.66) and that second hip fractures and major non-hip fractures requiring hospitalization had the same utility in the year of the event as those at the onset of hip fracture (0.46). These assumptions were varied in sensitivity analyses.

\section{Analysis}

Two cohorts of 1000 identical men and women were used to simulate a representative patient aged 83 years at hip fracture, with an average pre-admission Charlson comorbidity score of 1.2 and living in their own home before the fracture. We further assessed the cost-effectiveness of the models of care in several subgroups of hip fracture patients defined according to their age, sex, and Charlson comorbidity score at index hip fracture. A model of care was deemed to be cost-effective if the incremental costeffectiveness ratio (ICER) was below $£ 30,000$ per quality-adjusted life years (QALY) gained. ${ }^{(22)}$ The ICER was estimated by dividing the difference in mean costs by the difference in mean effects (life years and QALYs) for a given model of care compared with its next best alternative. Internal validity of the model was checked using sensitivity analysis (extreme values) and by comparing the model outputs with the data used to build the model. Model parameters and structural assumptions were evaluated in one-way and probabilistic sensitivity analysis and quantified using a costeffectiveness acceptability curve $\left(\right.$ CEAC) ${ }^{(23)}$ and analysis of covariance methods (ANCOVA) ${ }^{(24)}$ (see Supplemental Material). Key uncertainties in the model structure were identified when developing the conceptual framework.

\section{Results}

\section{Risk and cost equations}

The mean follow-up time of patients in the HES data set was 2.6 years (SD 2.5) after index hip fracture, and 84,717 patient-years of data were available to estimate the risk equations. The number of patients reporting second hip fracture and major non-hip fractures were 2206 and 1464, respectively (Supplemental Table S7). Supplemental Tables S8 and S9 report the risk models, and Supplemental Tables S10-12 report the cost models. These models allow simulating the natural history of individuals and costs as well as patient heterogeneity. For example, being female was associated with higher risk of second hip fracture, higher risk of major non-hip fracture, lower risk of mortality (Supplemental Table S9), lower risk of hospitalization, and lower hospital costs (Supplemental Table S10), adjusting for other covariates. Patients living in a care home were associated with a higher risk of major non-hip fracture, higher risk of mortality (Supplemental Table S9), higher risk of hospital admission, and higher hospital costs if admitted, as well as higher primary care costs in the year of the hip fracture (Supplemental Tables S10 and S11), adjusting for other covariates.

\section{Representative patient-male}

Table 2 reports the total life years, QALYs, and costs associated with each of the three models of care. For our male cohort of 1000 , the introduction of an OG and FLS would result in a reduction of 26 (95\% confidence interval [Cl] 17-35) and 19 (95\% Cl 9-29) deaths within 30 days of primary hip fracture, respectively, compared with usual care (Supplemental Table S13). Within 1 year of primary hip fracture, the reduction in deaths by introducing an OG and FLS, compared with usual care, would be 58 (95\% Cl 44-71) and 46 (95\% Cl 28-63), respectively. On average, over the lifetime of a patient, when compared with usual care, we would expect each patient to experience an increase of $0.18(95 \% \mathrm{Cl} 0.14-0.23)$ and 0.14 (95\% Cl 0.08-0.19) life years (undiscounted) spent in their own home if an OG or FLS were to be introduced, respectively.

Relative to usual care, the mean discounted health care and care home costs would be $£ 2610$ (95\% Cl £2109-£3166) and $£ 1975$ (95\% Cl £1265-£2591) higher when an OG or FLS were introduced, respectively. Higher care home costs accounted for $35 \%$ and $44 \%$ of the additional costs of FLS and OG, respectively. This was a result of increased longevity due to OG or FLS being introduced. The discounted average QALYs gained by male patients, relative to usual care, were $0.13(95 \% \mathrm{Cl} 0.09-0.16)$ and 0.10 (95\% Cl 0.06-0.14) if an OG or FLS were to be introduced, respectively.

At a $£ 30,000$ per QALY threshold, the most cost-effective model of care was introducing an orthogeriatrician, with an ICER of $£ 23,407 / \mathrm{QALY}$ (Table 3 ). There was considerable uncertainty regarding the comparison between OG and FLS models of care with statistically nonsignificant difference in costs and QALYs being $£ 635$ (95\% Cl $-£ 207$ to $£ 1496)$ and $0.03(95 \% \mathrm{Cl}-0.02$ to 0.07), respectively (Table 3 and Supplemental Fig. S3). However, the probability that introducing OG-led FLS was the most costeffective option was estimated at $69 \%$.

\section{Representative patient-female}

For our female cohort of 1000, mortality at 30 days and within 1 year was lower compared with the male cohort in the usual care (Supplemental Table S13). Hence, the introduction of an OG and FLS would result in a reduction of $16(95 \% \mathrm{Cl} 11-22)$ and 12 (95\% Cl 5-18) deaths within 30 days of primary hip fracture, respectively, compared with usual care. Within 1 year of primary hip fracture, the reduction in deaths by introducing an OG and FLS, compared with usual care, was 42 (95\% Cl 31-51) and 33 (95\% Cl 21-45), respectively. On average, over a lifetime, when compared with usual care, we would expect each female to experience an increase of $0.17(95 \% \mathrm{Cl} 0.13-0.21)$ and $0.13(95 \%$ $\mathrm{Cl} 0.08-0.18$ ) life years (undiscounted) spent in their own home if an OG or FLS were to be introduced, respectively.

The mean discounted health care and care home costs were $£ 2547$ (95\% Cl £1993-£3035) and £1909 (95\% Cl £1272-£2515) higher by introducing an OG and FLS, respectively, relative to usual care. As a result of longer longevity relative to males, care home costs now accounted for $41 \%$ and $51 \%$ of the additional costs of FLS and OG, respectively. The discounted average QALYs gained by female patients, relative to usual care, were $0.12(95 \% \mathrm{Cl} 0.09-0.15)$ and $0.09(95 \% \mathrm{Cl} 0.06-0.13)$ if an OG or FLS were to be introduced, respectively.

At a $£ 30,000$ per QALY threshold, the most cost-effective model of care for females was introducing an orthogeriatrician, with an ICER of $£ 22,709 / \mathrm{QALY}$ (Table 3). As with males, considerable uncertainty exists when comparing OG and FLS 
Table 2. Mean Discounted Costs and Outcomes of the Differing Models of Secondary Prevention Care

\begin{tabular}{|c|c|c|c|}
\hline & Usual care & Fracture liaison nurse & Orthogeriatrician \\
\hline Representative male ${ }^{a}$ & Mean $(95 \% \mathrm{Cl})$ & Mean $(95 \% \mathrm{Cl})$ & Mean $(95 \% \mathrm{Cl})$ \\
\hline Total costs & $\begin{array}{c}£ 39,069 \\
(£ 37,798-£ 40,514)\end{array}$ & $\begin{array}{c}£ 41,044 \\
(£ 39,495-£ 42,621)\end{array}$ & $\begin{array}{c}£ 41,679 \\
(£ 40,265-£ 43,262)\end{array}$ \\
\hline Intervention & 0 & $£ 200$ & $£ 420$ \\
\hline Hospital care & $\begin{array}{c}£ 23,025 \\
(£ 22,406-£ 23,679)\end{array}$ & $\begin{array}{c}£ 23,678 \\
(£ 22,988-£ 24,384)\end{array}$ & $\begin{array}{c}£ 23,814 \\
(£ 23,132-£ 24,525)\end{array}$ \\
\hline Primary care & $\begin{array}{c}£ 3,276 \\
(£ 3046-£ 3490)\end{array}$ & $\begin{array}{c}£ 3,471 \\
(£ 3234-£ 3714)\end{array}$ & $\begin{array}{c}£ 3,523 \\
(£ 3285-£ 3762)\end{array}$ \\
\hline Care home & $\begin{array}{c}£ 12,767 \\
(£ 11,893-£ 13,817)\end{array}$ & $\begin{array}{c}£ 13,695 \\
(£ 12,689-£ 14,837)\end{array}$ & $\begin{array}{c}£ 13,922 \\
(£ 12,962-£ 15,039)\end{array}$ \\
\hline Total life years & $\begin{array}{c}2.68 \\
(2.56-2.79)\end{array}$ & $\begin{array}{c}2.83 \\
(2.70-2.96)\end{array}$ & $\begin{array}{c}2.88 \\
(2.75-3.00)\end{array}$ \\
\hline Total QALYs & $\begin{array}{c}1.64 \\
(1.46-1.83)\end{array}$ & $\begin{array}{c}1.74 \\
(1.54-1.96)\end{array}$ & $\begin{array}{c}1.77 \\
(1.56-1.98)\end{array}$ \\
\hline \multicolumn{4}{|l|}{ Representative female ${ }^{a}$} \\
\hline Total costs & $\begin{array}{c}£ 50,534 \\
(£ 49,226-£ 52,276)\end{array}$ & $\begin{array}{c}£ 52,444 \\
(£ 50,935-£ 54,340)\end{array}$ & $\begin{array}{c}£ 53,081 \\
(£ 51,559-£ 54,974)\end{array}$ \\
\hline Intervention & 0 & $£ 200$ & $£ 420$ \\
\hline Hospital care & $\begin{array}{c}£ 23,893 \\
(£ 23,390-£ 24,471)\end{array}$ & $\begin{array}{c}£ 24,387 \\
(£ 23,804-£ 25,040)\end{array}$ & $\begin{array}{c}£ 24,478 \\
(£ 23,918-£ 25,073)\end{array}$ \\
\hline Primary care & $\begin{array}{c}£ 4,721 \\
(£ 4417-£ 5016)\end{array}$ & $\begin{array}{c}£ 4,902 \\
(£ 4571-£ 5224)\end{array}$ & $\begin{array}{c}£ 4,955 \\
(£ 4635-£ 5265)\end{array}$ \\
\hline Care home & $\begin{array}{c}£ 21,921 \\
(£ 20,972-£ 23,134)\end{array}$ & $\begin{array}{c}£ 22,955 \\
(£ 21,925-£ 24,301)\end{array}$ & $\begin{array}{c}£ 23,229 \\
(£ 22,191-£ 24,524)\end{array}$ \\
\hline Total life years & $\begin{array}{c}3.89 \\
(3.77-4.03)\end{array}$ & $\begin{array}{c}4.04 \\
(3.91-4.19)\end{array}$ & $\begin{array}{c}4.09 \\
(3.95-4.24)\end{array}$ \\
\hline Total QALYs & $\begin{array}{c}2.42 \\
(2.15-2.72)\end{array}$ & $\begin{array}{c}2.52 \\
(2.23-2.82)\end{array}$ & $\begin{array}{c}2.54 \\
(2.26-2.85)\end{array}$ \\
\hline
\end{tabular}

${ }^{\mathrm{a}}$ Aged 83 years at hip fracture, with an average pre-admission Charlson-comorbidity score of 1.2 and living in their own home before the fracture.

models of care with again nonsignificant differences in costs and QALYs: $£ 638(95 \% \mathrm{Cl}-£ 207$ to $£ 1,418)$ and $0.03(95 \% \mathrm{Cl}-0.02$ to 0.07 ), respectively (Table 3 and Supplemental Fig. S4). Finally, the probability that introducing OG-led service was the most cost-effective option was estimated at $72 \%$.
Sensitivity analysis

Fig. 3 reports the impact of uncertainty in different parameters on the uncertainty of incremental costs and QALYs of introducing an orthogeriatrician compared with FLS. This was

Table 3. Cost-Effectiveness of the Differing Models of Secondary Prevention Care of Hip Fractures

\begin{tabular}{|c|c|c|c|c|c|c|}
\hline & $\begin{array}{l}\text { Difference } \\
\text { in costs }\end{array}$ & $\begin{array}{l}\text { Difference } \\
\text { in LYs }\end{array}$ & $\begin{array}{l}\text { Difference } \\
\text { in QALYs }\end{array}$ & $\begin{array}{l}\text { ICER } \\
(£ / L Y)\end{array}$ & $\begin{array}{c}\text { ICER } \\
(£ / Q A L Y)\end{array}$ & $\begin{array}{c}\text { Probability that is the } \\
\text { most cost-effective } \\
\text { at } £ 30,000 / Q A L Y\end{array}$ \\
\hline Representative male & Mean $(95 \% \mathrm{Cl})$ & Mean $(95 \% \mathrm{Cl})$ & Mean $(95 \% \mathrm{Cl})$ & Mean & Mean & \\
\hline Usual care & - & - & - & - & - & $0 \%$ \\
\hline FLS versus usual care & $\begin{array}{l}£ 1,975(1297 \\
\text { to } 2620)\end{array}$ & $\begin{array}{l}0.159(0.095 \\
\text { to } 0.218)\end{array}$ & $\begin{array}{l}0.099(0.058 \\
\text { to } 0.140)\end{array}$ & $£ 12,458$ & $£ 19,955$ & $31 \%$ \\
\hline $\begin{array}{l}\text { OG versus fracture } \\
\text { liaison nurse }\end{array}$ & $\begin{array}{c}£ 635(-207 \\
\text { to } 1496)\end{array}$ & $\begin{array}{l}0.043(-0.031 \\
\quad \text { to } 0.116)\end{array}$ & $\begin{array}{l}0.027(-0.019 \\
\quad \text { to } 0.074)\end{array}$ & $£ 14,898$ & $£ 23,407$ & $69 \%$ \\
\hline \multicolumn{7}{|l|}{ Representative female } \\
\hline Usual care & - & - & - & - & - & $0 \%$ \\
\hline FLS versus usual care & $\begin{array}{l}£ 1,909(1271 \\
\text { to } 2562)\end{array}$ & $\begin{array}{c}0.149(0.094 \\
\text { to } 0.209)\end{array}$ & $\begin{array}{c}0.093(0.057 \\
\text { to } 0.133)\end{array}$ & $£ 12,837$ & $£ 20,421$ & $28 \%$ \\
\hline $\begin{array}{l}\text { OG versus fracture } \\
\text { liaison nurse }\end{array}$ & $\begin{array}{c}£ 638(-207 \\
\text { to } 1418)\end{array}$ & $\begin{array}{l}0.044(-0.032 \\
\quad \text { to } 0.110)\end{array}$ & $\begin{array}{l}0.028(-0.020 \\
\text { to } 0.071)\end{array}$ & $£ 14,618$ & $£ 22,709$ & $72 \%$ \\
\hline
\end{tabular}

$\mathrm{LY}=$ life years; $\mathrm{QALY}=$ quality-adjusted life years; ICER = incremental cost-effectiveness ratio; $\mathrm{FLS}=$ fracture liaison service; $\mathrm{OG}=$ orthogeriatric . 
largely associated with the uncertainty in the relative effectiveness of an OG-led model of care compared with FLS on 30-day (accounting for $25 \%$ of all uncertainty in incremental costs and QALYs) and 1-year mortality (52\% to $58 \%$ of all uncertainty) after index hip fracture. The uncertainty in the natural history components of the model (eg, absolute mortality, probability of second hip fracture, or other major fragility fracture) was associated with $10 \%$ of all uncertainty in the incremental costs and QALYs. In terms of model structural assumptions, we tested the impact of changes to these assumptions on the cost-effectiveness results (Supplemental Table S14). Overall, at a $£ 30,000 / Q A L Y$ threshold, the introduction of OG or FLS relative to usual care remained the most cost-effective options when the assumptions were changed. For example, assuming the effect of the interventions concerning second hip fracture extended over lifetime rather than 2 years resulted in OG becoming more cost-effective ( $£ 20,036 / \mathrm{Q} A L Y)$. Also, assuming that the effect of the interventions on mortality extended over lifetime rather than 1 year after hip fracture resulted in OG being more cost-effective ( $£ 18,052 / \mathrm{Q} A L Y)$. Excluding care home costs resulted in OG becoming more cost-effective (£13,039/QALY and £14,733/QALY for women and men, respectively). Assuming that the OG and FLS services resulted in additional $£ 1000$ per patient in management and test costs during the hip fracture admission relative to usual care resulted in OG becoming borderline cost-effective ( $£ 29,573 / \mathrm{QALY}$ ). Finally, using the smallest hospital in the UK region (220 hip fractures per year) to estimate the intervention cost per patient (OG at $£ 859$ per patient and FLS at $£ 409$ per patient) resulted in FLS becoming the most cost-effective option (ICER of $£ 22,922 / \mathrm{QALY}$; Supplemental Table S14).

\section{Subgroup analysis}

For patients up to age 80 years, introducing an OG was the most cost-effective option (Supplemental Table S15). In contrast, introducing an FLS became the most cost-effective option for

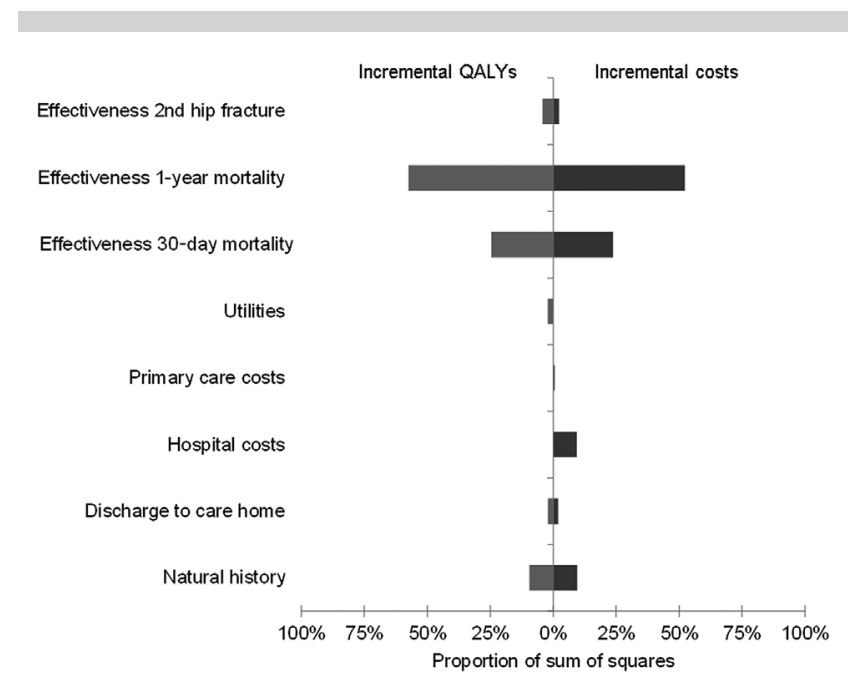

Fig. 3. Orthogeriatrician- versus nurse-led fracture liaison service: ANCOVA analysis of proportion of sum of squares for incremental QALYs saved and incremental costs explained by the uncertainty in the model. The horizontal axis represents the variation in incremental costs and QALYs that is associated with the uncertainty in the model inputs. patients aged 90 years if their Charlson comorbidity score at index hip fracture was 5 or above.

\section{Discussion}

Our cost-effectiveness analysis of models of hip fracture care found that the introduction and/or expansion of OG- and nurseled FLS was more effective and cost-effective than usual care.

Two recent systematic reviews assessed the economic evidence concerning the prevention of osteoporotic fractures. $^{(25,26)}$ Consistent with our findings, three previous economic evaluations, using Markov models, reported secondary fracture prevention interventions (ie, hospital osteoporosis case manager, outpatient-based FLS, and hospital nurse-led FLS) to be cost-effective in populations with fragility fracture. ${ }^{(12,27,28)}$ These analyses were limited by the use of data from single institutions and by a relatively short follow-up period (up for 1 year) or by the use of disparate data sources covering different populations of patients and time periods. Our study benefitted from the availability of large primary and hospital data sets to robustly estimate the impact of the models of care across a large representative population in terms of survival, prevention of second hip fracture, primary care and hospital care costs, and cost-effectiveness. For example, having incorporated into our modeling robust estimates of the time to second hip fracture and death and the short- and long-term costs associated with patients with hip fracture, we did not find the introduction and/ or expansion of OG- or nurse-led services to be cost-saving compared with usual care, in contrast to previous work. ${ }^{(12,27)}$ This is largely explained by the added longevity that translates into higher costs and refractures compared with usual care.

Irrespective of how patients were stratified in terms of their age, sex, and Charlson comorbidity score at index hip fracture, our results suggest that it is cost-effective to introduce an orthogeriatrician or FLS compared with usual care. We find that these models of care produce greater gains in life years than QALYs, suggesting that the positive impact on survival was not necessarily accompanied by proportional gains in quality of life. This is an area that would repay closer investigation.

There is considerable uncertainty in the evidence informing the model, particularly concerning the relative effectiveness of an OG- compared with a nurse-led FLS on survival and prevention of second hip fracture and also concerning the natural history of hip fracture. The large number of hip fractures every year for which these interventions are potentially relevant suggests that caution is required about decisions based on the model results. Our findings highlight the need for further research to reduce decision uncertainty, with particular emphasis on undertaking clinical trials to obtain unbiased comparisons of the different models of secondary care services.

The translation of our findings into other types of acute fragility fractures requiring inpatient care is possible. However, the management of vertebral fractures requires additional components, different mechanisms for case finding (routine VFA, text mining radiology reports, rereading axial imaging) as well as treatment (analgesia, vertebral augmentation) relative to hip fractures. Although these types of care pathways can be delivered within an FLS system, they require layering of additional work flows and remain a priority for future service development. $^{(29)}$

Furthermore, the case-mix of patients seen by the OG- and nurse-led FLS services may vary by type of fracture and frailty of 
patient; however, our qualitative evaluation of hip fracture services within the region did not detect differences in the types of patients that were seen by OG and FLS models of care. ${ }^{(14)}$

Our study had several limitations. First, although we improved on previous studies by basing our estimates of effectiveness on a very large administrative data set supported by a careful survey of the services provided in each hospital, ${ }^{(14,15)}$ there were no effectiveness data informed by clinical trials, and the limited range and number of services currently provided in England precluded considering some models of care, such as introducing a combined orthogeriatrician- and nurse-led FLS. Second, we did not separate the different types of fragility-related fractures that patients could suffer post-discharge. We focused on major nonhip fractures requiring hospitalization given their relative large impact in terms of health care costs. Furthermore, we did not separate non-hip fractures by type so that we could benefit from a larger sample to estimate the costs. Nonetheless, other fragility fractures not incurring hospitalization were still captured in the model in the primary and hospital care costs. Third, we did not have quality-of-life data from individual patient records and relied instead on a systematic review and meta-regression of published literature. This provided estimates of changes in EQ5D-based utility conditional on time since hip fracture, but we could not reliably estimate utility values for non-hip fractures or the additional impact these may have on the quality of life of individuals with a history of hip fracture. The consequent assumptions were explored in sensitivity analysis, but future research could address this limitation. Furthermore, the use of a cohort-based Markov model made it difficult to capture the trajectory of utility values taking into account both time since primary hip fracture and history of events such as non-hip fractures and second hip fracture. An individual-based (ie, microsimulation) Markov model would have facilitated tracking of each individual's history and time-varying utility values, but would have been more time-consuming to construct and computationally intensive to run. ${ }^{(30)}$ Finally, we only included health care and care home costs, and so excluded some important economic considerations for people with hip fracture and their families, such as unpaid care provided by friends and family, walking aids, and home adaptation costs as a consequence of the fracture, as well as locally funded home social care (eg, provision of meals, nursing care, live-in help, etc.). Future research using UK-based populations of hip fracture patients is needed to assess the use and costs of these resources as well as the impact on informal care.

In conclusion, our work suggests that it is cost-effective to introduce an orthogeriatrician or FLS secondary care service for patients with a hip fracture, predominantly because of their effects on mortality rather than on refracture. Further research is needed to make more informed decisions with a focus on estimating the effectiveness of these models of care informed by clinical trials.

\section{Disclosures}

JL, AM, DPA, NKA, CC, MKJ, and AJ received grants from NIHR HS\&DR during the conduct of the study. Outside the submitted work, MKJ reports personal fees from Lilly UK, Amgen, Servier, Merck, Medtronic, Internis, and Consilient Health and serves on the Scientific Committee of the National Osteoporosis Society and International Osteoporosis Foundation. DPA received grants from Bioiberica S.A. and Amgen Spain S.A. CC received personal fees from Servier, Amgen, Eli
Lilly, Merck, Medtronic, and Novartis. NKA reports personal fees from Merck, Smith and Nephew, Q-Med, Nicox, Flexion, Bioiberica, and Servier and grants and personal fees from Roche. AJ has received consultancy, lecture fees, and honoraria from Servier, UK Renal Registry, Oxford Craniofacial Unit, IDIAP Jordi Gol, and Freshfields Bruckhaus Deringer, has held advisory board positions (which involved receipt of fees) from Anthera Pharmaceuticals, Inc., and received research sponsorship from Roche. SH and AD state that they have no conflicts of interest.

\section{Acknowledgments}

This project was funded by the NIHR Health Services and Delivery Research programme (project number 11/1023/01). Support was received from the Oxford NIHR Musculoskeletal Biomedical Research Unit, Nuffield Orthopaedic Centre, University of Oxford. The views and opinions expressed therein are those of the authors and do not necessarily reflect those of the HS\&DR programme, NIHR, NHS, or the Department of Health. This study is based in part on data from Hospital Episode Statistics and the Clinical Practice Research Datalink obtained under license from the UK Medicines and Healthcare Products Regulatory Agency. However, the interpretation and conclusions contained in this study are those of the authors alone. The ReFRESH study group consists of Dr Andrew David Judge, Dr Muhammad Kassim Javaid, Professor Nigel Arden, Professor Cyrus Cooper, Professor Andrew Farmer, Dr Daniel PrietoAlhambra, Dr Jose Leal, Professor Michael Goldacre, Professor Alastair Gray, Dr Janet Lippett, Dr Rachael Gooberman-Hill, and Laura Graham.

Authors' roles: All authors had full access to all statistical reports and tables in the study. JL takes responsibility for the integrity and accuracy of the economic analysis. All authors revised and approved the final version of the article. AJ and MKJ supervised the study and are joint senior authors.

\section{References}

1. Abrahamsen B, van Staa T, Ariely R, Olson M, Cooper C. Excess mortality following hip fracture: a systematic epidemiological review. Osteoporos Int. 2009;20(10):1633-50.

2. Cooper $C$, Mitchell $P$, Kanis JA. Breaking the fragility fracture cycle. Osteoporos Int. 2011;22 (7):2049-50.

3. Hernlund $E$, Svedbom A, Ivergard $M$, et al. Osteoporosis in the European Union: medical management, epidemiology and economic burden. A report prepared in collaboration with the International Osteoporosis Foundation (IOF) and the European Federation of Pharmaceutical Industry Associations (EFPIA). Arch Osteoporos. 2013;8(1-2):136.

4. Leal J, Gray AM, Prieto-Alhambra D, et al. Impact of hip fracture on hospital care costs: a population-based study. Osteoporos Int 2016;27(2):549-558.

5. Marsh D, Akesson K, Beaton DE, et al. Coordinator-based systems for secondary prevention in fragility fracture patients. Osteoporos Int. 2011;22(7):2051-65.

6. Eisman JA, Bogoch ER, Dell R, et al. Making the first fracture the last fracture: ASBMR task force report on secondary fracture prevention. J Bone Miner Res. 2012;27(10):2039-46.

7. Department of Health. Falls and fractures: effective interventions in health and social care. 2009. Available at: http://www.dh.gov. uk/prod_consum_dh/groups/dh_digitalassets/@dh/@en/@pg/ documents/digitalasset/dh_109122.pdf.

8. Department of Health. Prevention package for older people. Available at: www.dh.gov.uk/en/Publicationsandstatistics/Publications/dh_103146. 
9. British Orthopaedic Association. The care of patients with fragility fracture. Available at: http://wwwfracturescom/pdf/BOA-BGS-BlueBook.pdf.

10. National Institute for Health and Care Excellence. Hip fracture: the management of hip fracture in adults. NICE guidelines CG124. Available at: https://www.nice.org.uk/guidance/cg124.

11. NOS and AGE UK. Report to the Minister of State for Care Services: breaking through: building better falls and fracture services in England. Available at: https://www.nos.org.uk/documentdoc?id=987.

12. McLellan AR, Wolowacz SE, Zimovetz EA, et al. Fracture liaison services for the evaluation and management of patients with osteoporotic fracture: a cost-effectiveness evaluation based on data collected over 8 years of service provision. Osteoporos Int. 2011; 22(7):2083-98

13. Royal College of Physicians. Falling standards, broken promises: report of the National Audit of Falls and Bone Health in Older People 2010. Available at: https://www.nos.org.uk/document.doc? id $=1516$

14. Drew S, Sheard S, Chana J, Cooper C, Javaid MK, Judge A. Describing variation in the delivery of secondary fracture prevention after hip fracture: an overview of 11 hospitals within one regional area in England. Osteoporos Int. 2014;25(10):2427-33.

15. Hawley S, Javaid MK, Prieto-Alhambra D, et al. Clinical effectiveness of orthogeriatric and fracture liaison service models of care for hip fracture patients: population-based longitudinal study. Age Ageing. 2016;45(2):236-42.

16. Barendregt JJ. The life table method of half cycle correction: getting it right. Med Decis Making. 2014;34(3):283-5.

17. Department of Health. NHS reference costs 2012 to 2013. Available at: https://www.gov.uk/government/publications/nhs-referencecosts-2012- to-2013.

18. Curtis LH. Unit costs of health and social care 2013. Personal Social Services Research Unit; 2013.

19. Health and Social Care Information Centre. Prescription cost analysis, England. 2013
20. National Hip Fracture Database. National report 2013. Available at: www.nhfd.co.uk.

21. Hayes AJ, Leal J, Gray AM, Holman RR, Clarke PM. UKPDS outcomes model 2: a new version of a model to simulate lifetime health outcomes of patients with type 2 diabetes mellitus using data from the 30-year United Kingdom Prospective Diabetes Study: UKPDS 82. Diabetologia. 2013;56(9):1925-33.

22. National Institute for Health and Care Excellence. Guide to the methods of technology appraisal 2013. Available at: http:// publications.nice.org.uk/pmg9.

23. Fenwick E, Claxton K, Sculpher M. Representing uncertainty: the role of cost-effectiveness acceptability curves. Health Econ. 2001;10(8): 779-87.

24. Briggs $A$, Sculpher $M$, Claxton K. Decision modelling for health economic evaluation. Oxford, UK: Oxford University Press 2006

25. Si L, Winzenberg TM, Palmer AJ. A systematic review of models used in cost-effectiveness analyses of preventing osteoporotic fractures. Osteoporos Int. 2013;25(1):51-60.

26. Ganda K, Puech M, Chen JS, et al. Models of care for the secondary prevention of osteoporotic fractures: a systematic review and metaanalysis. Osteoporos Int. 2013;24(2):393-406.

27. Majumdar SR, Lier DA, Beaupre LA, et al. Osteoporosis case manager for patients with hip fractures: results of a cost-effectiveness analysis conducted alongside a randomized trial. Arch Intern Med. 2009; 169(1):25-31.

28. Cooper MS, Palmer AJ, Seibel MJ. Cost-effectiveness of the Concord Minimal Trauma Fracture Liaison Service: a prospective, controlled fracture prevention study. Osteoporos Int. 2012;23(1):97-107.

29. Javaid MK, Kyer C, Mitchell PJ, et al. Effective secondary fracture prevention: implementation of a global benchmarking of clinical quality using the IOF Capture the Fracture(R) Best Practice Framework tool. Osteoporos Int. 2015;26(11):2573-8.

30. Siebert U, Alagoz O, Bayoumi AM, et al. State-transition modeling: a report of the ISPOR-SMDM Modeling Good Research Practices Task Force-3. Value Health. 2012;15(6):812-20. 